\title{
DE OBSERVACIONES A ESTUDIOS. TÍTULOS DE LOS ARTÍCULOS CIENTÍFICOS ESPAÑOLES ENTRE 1799 Y 1920
}

FROM OBSERVATIONS TO STUDIES. TITLES OF SPANISH SCIENTIFIC ARTICLES BETWEEN 1799 AND 1920

\section{Dorota Kotwica}

University of Krákow

Resumen

En el presente artículo, se analizan algunas características de los artículos publicados por las instituciones científicas españolas entre 1799 y 1920. En concreto, se analizan las palabras descriptivas del tipo de texto presentes en los títulos, por ejemplo, estudio, memoria, análisis, ensayo, etc. La presencia de este tipo de «etiquetas» en los títulos es un rasgo común de muchos de los artículos publicados entre las fechas señaladas. Creemos que, por una parte, estas etiquetas reflejan la conciencia de los miembros de la comunidad científica sobre las convenciones de escritura científica. Por otra parte, ofrecen pistas sobre la evolución de las prácticas científicas a lo largo de este periodo.

Palabras Clave: artículo científico, revistas científicas, historia de la ciencia, siglo XIX, títulos.
Abstract

In this paper we analyze some features of scientific articles published in Spanish scholarly institutions between 1799 and1920. In particular, we analyze introductory words in the titles, such as study, memory, analysis, essay, etc. The presence of such «labels» in the titles was quite common in the articles published in that period. On the one hand, their use is a proof of how aware the scientific community members were about the conventions in scientific writing. On the other hand they offer insight into the evolution of writing practices in the period under review.

KEY WORDS: scientific article, scientific journals, history of science, $19^{\text {th }}$ century, titles 


\section{INTRODUCCIÓN}

Los estudios internacionales sobre la historia del género del artículo científico coinciden en ubicar el origen de este género en la segunda mitad del siglo XVII. Como apuntan McClellan (1979), Swales (2007 [1990]) y Gross, Harmon y Reidy (2002), el contexto de la gestación del artículo científico y su posterior evolución fueron las publicaciones periódicas editadas en las instituciones y sociedades científicas creadas entre los siglos XVII y XIX. De manera análoga a la situación descrita para otras lenguas, es lícito sospechar que la evolución de este género en el contexto español tuvo lugar en las páginas de Anales, Memorias y Revistas publicados por distintas instituciones de la ciencia. Según indican estudios históricos, es allí donde se reflejan los avances de la ciencia española y donde transcurre una parte importante de la comunicación científica entre los siglos XVIII y XIX. Sin embargo, hasta donde sepamos, estas publicaciones no se habían estudiado desde una óptica filológica o lingüística.

Nuestro objetivo en el presente trabajo es ofrecer una aproximación a los textos publicados entre 1799 y 1920 en las revistas científicas de España. Se trata de una primera exploración realizada en las revistas, a partir de los índices de las mismas y los títulos de textos que se incluyen. Pretendemos descubrir qué tipo de pistas proporcionan los índices y el formato de los títulos de los artículos acerca de la evolución de la ciencia y la estandarización de las convenciones de la escritura científica. Trabajos como los de Gross y otros (2002) demostraron el interés que tiene el estudio histórico de diversos aspectos del formato de los artículos científicos, incluidos los títulos. Asimismo, los resultados de estos estudios pueden ofrecer pistas sobre algunas tendencias en la evolución del género.

\section{PERIODISMO CIENTíFICO ENTRE LOS SIGLOS XVII Y XIX. LAS TENDENCIAS EUROPEAS Y EL CASO DE ESPAÑA}

Como se ha apuntado más arriba, los estudios clásicos sobre la evolución del género del artículo científico (McClellan, 1979; Swales, 2007 [1990]; Gross y otros, 2002) ubican sus orígenes en la segunda mitad del siglo XVII y relacionan su nacimiento con las primeras publicaciones de carácter periódico ${ }^{1}$ editadas por instituciones y sociedades científicas creadas en ese periodo, como La Académie de Sciences de Paris o Royal Society de Londres. Estas publicaciones permitían transmitir con rapidez nuevos descubrimientos realizados en diversos campos de la ciencia por medio de ensayos y cartas escritos por y para los miembros de la comunidad científica (Gross y otros, 2002: I). Diversos estudios han constatado que, entre los siglos XVII y XX, el género del artículo científico en inglés, francés o alemán experimentó una dinámica evolución que afectó todas las dimensiones del género, desde el lenguaje utilizado y el estilo, hasta el formato externo (McClellan, 1979; Bazerman, 1988; Gross y otros, 2002; Valle, 2004; Banks, 2008; etc.). Según Gross y otros (2002), en esta evolución, se aprecia una búsqueda constante de alcanzar la óptima eficacia en la consecución de los objetivos del autor, dentro del cambiante contexto histórico de la ciencia:

\footnotetext{
Just as with biological features, the selection of new communicative and argumentative features over time takes on a logical, almost inevitable appearance only in retrospect. The scientific article has evolved, not in the sense of becoming better (or worse), but in the sense of changing to cope with
}

\footnotetext{
1 En los trabajos sobre la historia del periodismo en inglés el término utilizado para referirse a estas publicaciones suele ser periodical. En la literatura española suelen utilizarse los términos de publicaciones periódicas (González González 1999, Corell Domenéch y Navarro Brotons 2004) y revistas (Bosch Carrera 1992, Algaba 2000, Corell Domenéch y Navarro Brotons 2004). En este trabajo, al referirnos a las publicaciones institucionales y de las sociedades científicas, optamos por utilizar indistintamente ambas denominaciones. 
the communicative and argumentative needs of an evolving set of disciplines whose messages have become ever more complex and have, consequently, strained to the utmost the resources inherent in natural languages (Gross y otros, 2002: 219).

En el caso de España, las cuestiones relacionadas con el periodismo especializado y con la evolución del género del artículo científico han recibido poca atención (Correll Doménech y Navarro Brotons, 2004). Desde el punto de vista de la historia externa, López Piñero y Terrada (1990) señalan el siglo XVIII como la «prehistoria del periodismo científico». Algaba (2000) relaciona el retraso editorial de este siglo con el carácter absolutista de la política nacional de aquel momento y con falta de apoyo suficiente por parte del gobierno. Los primeros ejemplos de publicaciones de instituciones científicas ${ }^{2}$ españolas comparables con sus precursoras europeas que hemos mencionado arriba se encuentran en la última década del siglo XVIII, por ejemplo, Anales del Real Laboratorio de Química de Segovia de Louis Proust (1792, 1795), Anales de Historia Natural (1799-1804) editado en el Real Gabinete de Historia Natural y Almanaque Náutico y Efemérides astronómicas (1791) publicado en el Observatorio de San Fernando (Correll Doménech y Navarro Brotons, 2004: 62). Con todo, el siglo XVIII no ofrece demasiados antecedentes de revistas científicas especializadas, en el sentido de revistas dedicadas íntegramente a contenidos científicos ${ }^{3}$ (Ten Ros y Celi Aragón, 1996: 16). Las iniciativas institucionales del fin del siglo XVIII se truncaron a causa de los acontecimientos del primer tercio del siglo XIX. Según López Piñero (1992), la situación de la ciencia en general mejoró durante el periodo isabelino (1834-1868) y, de acuerdo con Algaba (2000), en el mismo periodo tuvo lugar el «despegue del periodismo científico", seguido por una etapa de consolidación posterior a la Revolución de 1868.

A lo largo del siglo XIX, los artículos relacionados con la ciencia aparecían en publicaciones divulgativas, culturales y generalistas, mientras que las revistas científicas especializadas eran escasas (Corell Doménech y Navarro Brotons, 2004: 62). Entre ellas, destacaban publicaciones editadas por instituciones científicas como la Sociedad Española de Historia Natural (1871), la Academia de Ciencias Naturales y Artes en Barcelona (1764) o la Real Academia de Ciencias Exactas, Físicas y Naturales (1847). Se observa, por tanto, que la historia de la comunicación científica en España ente los siglos XVIII y XIX repite el modelo inaugurado por otras naciones en el que las revistas especializadas son fruto de la labor de instituciones y sociedades científicas.

En definitiva, a pesar de la escasez de datos sobre la evolución del periodismo científico en España, se pueden intuir algunas tendencias análogas a las corrientes europeas entre los siglos XVII y XX. En concreto, postulamos que el periodismo científico especializado, y por tanto, el género del artículo científico, se gestó en España en las revistas de las instituciones y las sociedades científicas.

\section{$3 \quad$ TítUlos dE LOS ARTÍCULOS CIENTíFICOS}

Es necesario recordar que la manera en que se comunica la ciencia ha variado en el tiempo (Hyland, 2009) y que, asimismo, los géneros científicos son susceptibles de variación diacrónica (Swales, 2004; Parodi, Ibáñez y Venegas, 2009). Ambos tipos de variación se pueden apreciar en la evolución de diversos rasgos del discurso científico, por ejemplo, el uso de la terminología, los modelos de construcción sintáctica, los elementos de la

\footnotetext{
2 Fuera del ámbito de la medicina, cuya evolución no se tratará aquí.

${ }^{3}$ En cambio, a lo largo del siglo XVIII, existían algunas publicaciones de carácter general que dedicaron algún espacio a la información relativa a la ciencia, por ejemplo, Efemérides barométrico-médicas matritenses (1734), Memorias eruditas para la crítica de arte y ciencia (1736), Diario de los Literatos de España (1737). (Corell Doménech y Navarro Brotons, 2004: 60) Normas (ISSN: 2174-7245) | 
argumentación, o, incluso, los rasgos más superficiales como la presencia de elementos visuales, títulos y división en apartados. Nuestro estudio se interesa en los rasgos comprendidos en este último grupo, en concreto, los cambios relativos al formato de los títulos de textos.

Gross y otros (2002) observan una evolución en cuanto a los títulos, tanto en el título principal del artículo como en los títulos de los apartados y las partes del texto. Apuntan que entre los siglos XVIII y XX, el título principal del artículo experimenta varios cambios. Por ejemplo, los títulos del XVIII solían ser temáticos, pero no incluían terminología; el uso de esta aumentó visiblemente en los títulos del siglo XIX. Además, los mencionados autores observan un rasgo sintáctico común para todo el periodo y para las tres lenguas analizadas (inglés, francés y alemán), esto es, la inclusión de alguna preposición que introduce el tema del texto (concerning, sur, über, etc.) (Gross y otros, 2002). Gross y otros (2002) no profundizan sobre una característica peculiar de los títulos de los artículos que ellos mismos citan, por ejemplo, "An Account of an Experiment Made before the Royal Society, Touching the Proportion of the Weight of Air, to the Weight of a like Bulk of Water, Without Knowing the Quantity of Either» (1705, inglés), "Various Observations Concerning the Raising of Bees» (1754, francés), "Note on the Hypotrophy of the Spleen and the Alteration of the Blood, Consisting of a Rise in the White Corpuscules» (1854, francés). Y es que todos los títulos citados, aparte de la preposición tematizadora, incluyen un elemento descriptivo (que hemos marcado con negrita): «account», «observations», «note». Este tipo de elementos son los que nos interesan en el presente trabajo.

En el presente estudio nos centramos en estas palabras descriptivas del tipo de texto y, en lo que sigue, las denominamos etiquetas. Se podría pensar, incluso, que se trata de indicadores de ciertos tipos de textos o incluso (sub)géneros. Sin embargo, no pretendemos equiparar las etiquetas con la nomenclatura de géneros, puesto que somos conscientes de los problemas que esto conllevaría. Si bien, según Swales (2007 [1990]: 54-55), los nombres de géneros utilizados dentro de una comunidad discursiva proporcionan información sobre los mismos, deben ser tratados con cautela y contrastados con el objetivo real que se persigue en cada evento comunicativo. En ocasiones, estos nombres pueden ser simplemente etiquetas preestablecidas por las normas de alguna institución. Además, las nomenclaturas también experimentan variación histórica.

De todas formas, los títulos de los artículos científicos ofrecen muestras de una reflexión metalingüística de los autores acerca del tipo de actividad que realizan en el texto. Asimismo, pueden ser vistos como muestras de sus conocimientos acerca de las prácticas de la comunicación científica en el periodo analizado, pues, como apunta Swales (2007 [1990]: 54-55), los miembros más activos de la comunidad científica son los que mejor conocen las convenciones del género y quienes asocian ciertas acciones retóricas recurrentes con un género concreto.

En el apartado siguiente, mostramos alunas de las «prescripciones» institucionales vigentes en el periodo de estudio que contienen alguna información sobre el tipo de artículos que se publicaban en sus revistas científicas. 


\title{
4 TIPOS DE TEXTOS PUBLICADOS EN LAS REVISTAS CIENTÍFICAS ESPAÑOLAS SEGÚN LOS DOCUMENTOS INSTITUCIONALES
}

La información sobre el tipo de textos que se publicarían en las páginas de las revistas científicas españolas del periodo analizado suele encontrarse en los documentos que aportan información sobre la institución y el proyecto editorial de sus fundadores (estatutos, reglamentos, prólogos, etc.). En el prólogo al primer tomo de Anales de Historia Natural (AHN) (1799-1804), revista publicada por el Gabinete de Historia Natural, se informa de que la revista pretendía nutrirse del ejemplo de otras «naciones cultas» y publicar descubrimientos:

\begin{abstract}
Deseando el Rey, á exemplo de otras naciones cultas, se publique en sus estados un Periódico, que no solo presente a los nacionales los descubrimientos hechos y que vayan haciendo los extrangeros, sino tambien los que sucesivamente se hacen en España en la Mineralogía, Química, Botánica y otros ramos de Historia natural, ha resuelto S. M. confiar á D. Christiano Herrgen, D. Luis Proust, D. Domingo Fernandez y D. Antonio Josef Cavanilles la redaccion de esta importante obra, que se imprimirá en su Real imprenta baxo el nombre de Anales de Historia natural4. («Prólogo», AHN, l/1 (1799)5, la negrita es nuestra)6
\end{abstract}

Unos años más tarde, en 1801, con el cambio del título de la publicación a Anales de Ciencias Naturales ( $A C N$ ), en una "Advertencia» se invita a publicar en la revista los descubrimientos y también las memorias:

\begin{abstract}
El título de Historia natural que dimos á estos Anales nos pareció el debido para empezar á reunir en un cuerpo los descubrimientos nacionales y extranjeros, hasta que un mayor número de memorias pudiese ampliar los estrechos límites prefijados al principio. Conociamos el enlace que reina entre las ciencias, y los socorros mutuos que se prestan; y esperábamos que los profesores de aquellas que con miras diversas tratan de la naturaleza, y contribuyen á la ilustración general, depositarian sus descubrimientos en nuestros Anales. Habiéndose realizado estas justas esperanzas, mudamos el antiguo título de esta obra en el de Anales de Ciencias naturales, para que sin contravenir á él podamos publicar lo perteneciente á la historia natural, y á las ciencias que por qualquier título tratan de la naturaleza. Con este motivo convidamos de nuevo á los sabios nacionales y extrangeros para que nos comuniquen sus descubrimientos, que se imprimirán con el nombre de su respectivo autor. ("Advertencia», ACN, III/7, (1801), la negrita es nuestra)
\end{abstract}

Según se desprende de esta advertencia, se consideraba que los Anales contenían descubrimientos descritos en formato de memorias, por lo que se puede sospechar que la memoria fue el prototipo de texto publicado en esta revista.

Por su parte, en los «Estatutos» de la Real Academia de Ciencias (RAC) (publicados en el primer tomo de las Memorias, en 1850), se establece una lista más exhaustiva de tipos de textos publicados bajo sus auspicios:

La Academia podrá publicar:

$1 .^{\circ}$ El resumen anual de sus tareas:

2. Las memorias é informes de los académicos y de las comisiones:

3. ${ }^{\circ}$ Las memorias premiadas:

4. Las memorias de la extinguida Academia de Ciencias naturales:

\footnotetext{
${ }^{4}$ Este fragmento del «Prólogo» es en realidad una cita del decreto expedido por el gobierno.

${ }^{5}$ Acceso en línea en: http://bibdigital.rjb.csic.es/spa/Libro.php?Libro=738

${ }^{6}$ Todos los textos se citan conservando la ortografía original.

${ }^{7}$ Acceso en línea: http://bibdigital.rjb.csic.es/ing/Libro.php?Libro=740 
5. Las memorias ó cualesquiera otros escritos presentados á la Academia por personas extrañas á la Corporación, prévio su consentimiento:

6. ' Los elogios fúnebres de los académicos. («Estatutos», MemoRAC, I (1850), p. XXXI, la negrita es nuestra) ${ }^{8}$

Las Memorias de la RAC se pensaron para incluir sobre todo distintos tipos de memorias $u$ otros escritos que se le ofrecieran. Además, se publicaban también discursos pronunciados en las reuniones anuales de los académicos (según los «Estatutos», MemoRAC, I (1850), XVI-XVII).

Por su parte, en la «Circular» de 1872, la Sociedad Española de Historia Natural, aporta una lista de tipos de trabajos que incluirían sus Anales (ASEHN):

\begin{abstract}
Tan importante objeto tendrán los Anales de la Sociedad española de Historia Natural, y en ellos se insertarán preferentemente los catálogos totales ó parciales de las producciones de una localidad determinada, la descripcion de especies nuevas, la crítica de las ya publicadas, é igualmente las monografías de un grupo particular de séres naturales, cuando haya suficientes datos para ello, y las noticias parciales acerca de la gea, flora y fauna de la Península y sus provincias ultramarinas, todo acompañado de los grabados y láminas necesarias. ("Circular», ASEHN, I (1872), p. VI, la negrita es nuestra) ${ }^{9}$
\end{abstract}

Según se desprende de otros fragmentos de esta circular, el nombre genérico bajo el que sus autores consideraban los textos incluidos en los anales fue memorias: "Las opiniones emitidas en las Memorias publicadas en los ANALES son de la exclusiva responsabilidad de sus autores» ( $p$. IV); aunque también se refieren a ellas de modo más genérico como «trabajos» (sin marcar la palabra con mayúscula, como se hace en el caso de Memorias), por ejemplo: «Los autores de las Memorias publicadas podrán obtener una tirada aparte de su trabajo,» (p. XI), «(n)ingún trabajo se puede insertar en los ANALES sin haber sido leido ántes en la Sociedad (...)» (p. XIV).

Los editores de la otra publicación de la misma Sociedad, las Memorias, se señala como objetivos de su publicación diversos tipos de estudios, trabajos, monografías y catálogos:

\begin{abstract}
Las MEMORIAS aparecerán por tomos completos con numeración correlativa, comprendiendo necesariamente estudios sobre los tres grupos de seres naturales, siempre que hubiere materiales para ello; y, tendrán cabida en ellas, los estudios generales sobre Biología, los descriptivos y organográficos, los trabajos sinópticos y monografías, los necrológicos y bibliográficos y los catálogos de las producciones naturales de la Península Ibérica y Baleares (estos últimos con paginación independiente) («Reglamento de la Sociedad...», I (1901), p. 8, la negrita es nuestra) ${ }^{10}$
\end{abstract}

El mismo Reglamento hace referencia a los textos propuestos para la publicación como trabajos, de manera genérica («Los trabajos que deban ir acompañados de láminas ó grabados» p. 8), mientras que la palabra memoria(s) queda reservada, por lo que parece, para referirse al título de la publicación.

En resumen, se observa que los editores de las publicaciones científicas españolas tenían ideas bastante claras sobre qué tipo de textos aspiraban a publicar. Asimismo, los autores que querían publicar en ellas contaban con algunas indicaciones acerca de qué tipo de trabajos podrían presentar.

\footnotetext{
${ }^{8}$ Acceso en línea:http://www.biodiversitylibrary.org/item/30707\#page/39/mode/1up

${ }^{9}$ Acceso en línea: http://bibdigital.rjb.csic.es/spa/Libro.php?Libro=1103

${ }^{10}$ Acceso en línea: http://bibdigital.rjb.csic.es/ing/Libro.php?Libro=1182 


\section{SOBRE ESTE ESTUDIO}

Como se había indicado anteriormente, el presente trabajo abarca el periodo entre 1799 y 1920, sin embargo, los datos seleccionados no cubren todo este periodo de manera uniforme. Esto se debe a la situación externa de la ciencia española en el periodo de estudio, especialmente, las lagunas editoriales a principios del siglo XIX. A pesar de estas deficiencias en cuanto a la periodización que, como se ha explicado, son justificadas por el contexto histórico que manejamos, creemos que el presente trabajo, aunque no exhaustivo, supone una aportación válida al estudio de la historia del género del artículo científico en España.

En cuanto a los datos consultados, para el análisis de los índices y de los títulos se han seleccionado diversos tomos de cinco revistas correspondientes a tres instituciones científicas mencionadas ya anteriormente (Gabinete de Historia Natural, Sociedad Española de Historia Natural y Real Academia de Ciencias). La selección de los títulos concretos se ha hecho atendiendo a su relevancia para el desarrollo de la ciencia natural, especialmente, las ciencias biológicas:

\begin{tabular}{|l|l|l|l|l|}
\hline Institución & Publicación & $\begin{array}{l}\text { Periodo } \\
\text { incluido }\end{array}$ & $\begin{array}{l}\text { Número de } \\
\text { tomos } \\
\text { incluidos }\end{array}$ & $\begin{array}{l}\text { Número de } \\
\text { títulos } \\
\text { incluidos }\end{array}$ \\
\hline $\begin{array}{l}\text { Gabinete de } \\
\text { Historia Natural }\end{array}$ & AHN/ACN & $1799-1804$ & 7 & 178 \\
\hline $\begin{array}{l}\text { Real Academia } \\
\text { de Ciencias }\end{array}$ & MemoRAC & $1850-1861$ & 5 & 59 \\
\hline $\begin{array}{l}\text { Sociedad } \\
\text { Española de } \\
\text { Historia Natural }\end{array}$ & ASEHN & $1872-1902$ & 16 & 145 \\
\hline $\begin{array}{l}\text { Sociedad } \\
\text { Española de } \\
\text { Historia Natural }\end{array}$ & MemoSEHN & $1903-192011$ & 6 & 46 \\
\hline $\begin{array}{l}\text { Real Academia } \\
\text { de Ciencias }\end{array}$ & RevRAC & $1904-1920$ & 9 & 209 \\
\hline
\end{tabular}

\section{Tabla 1. Relación de los datos analizados}

Las revistas se han consultado en su versión digitalizada disponible en alguno de los siguientes repositorios en línea: la Biblioteca Digital del Real Jardín Botánico CSIC ${ }^{12}$, Internet Archive $^{13}$ y Biodiversity Heritage Library ${ }^{14}$.

\footnotetext{
11 Las fechas finales en el caso de MemoSEHN y RevRAC se refieren al periodo comprendido en este estudio, no fechas finales de publicación de estas revistas.

${ }_{12}$ Acceso en línea: http://bibdigital.rjb.csic.es/spa/index.php

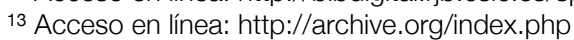

${ }^{14}$ Acceso en línea: http://www.biodiversitylibrary.org/ 
Para asegurar cierta homogeneidad de los datos, en cuanto al tipo de textos cuyos títulos se incluyen, hemos realizado una selección de textos previa al análisis, de acuerdo con los siguientes criterios:

- Se han tenido en cuenta solamente los títulos escritos en español. Asimismo, se han descartado textos escritos originalmente en otras lenguas.

- Se han incluido los títulos que comprenden la parte central de la revista, es decir, la parte dedicada a la presentación de trabajos originales, estudios y aportaciones de interés principalmente científico. Por el contrario, se han descartado Actas o anejos de las revistas, de contenidos más dispersos.

En cuanto a la manera de contabilizar los títulos, si un mismo trabajo (bajo el mismo título) aparecía fragmentado en varias partes, se ha contabilizado como un título. Cabe advertir también que los resultados del análisis se refieren a los títulos en la versión proporcionada por el índice. Esto resulta especialmente relevante para los textos más tempranos que en muchas ocasiones se publicaban con títulos modificados respecto a la versión presentada en el índice.

Dentro de los índices, hemos centrado nuestra atención en la presencia de palabras que encabezan los títulos y que de manera descriptiva aluden al carácter del artículo, es decir, las etiquetas. En los siguientes títulos hemos marcado con negrita ejemplos de etiquetas: "Ensayo monográfico sobre los Quirópteros de España», "Contribuciones a la flora de la Península ibérica", "Estudio de los caracteres métricos y morfológicos del cuerpo de los peces». A partir de la exploración de los índices, hemos elaborado una lista con todas las etiquetas encontradas y su frecuencia. Hemos tratado de observar qué relación existe entre las etiquetas utilizadas en los títulos y los tipos de textos mencionados en los documentos institucionales. Además, hemos realizado algunas consultas lexicográficas para observar la presencia acepciones "científicas» en las definiciones de ciertas etiquetas en los diccionarios de la época.

\section{RESULTADOS}

\subsection{Presencia de las etiquetas en los títulos}

El primer resultado del análisis de los índices es que las etiquetas no están presentes en todos los artículos. En los títulos sin etiquetas, el tema de artículo se introduce directamente sin ninguna palabra que señale el carácter de texto, por ejemplo: «Las plantas de Cartagena», «El fondo del mar entre la Península y Canarias», etc.

Por su parte, cuando aparece una etiqueta, esta puede ser simple (análisis, estudio, examen, clasificación, descripción, investigaciones, viaje, excursión, enumeración, catálogo, dato, experiencias, experimentos, indagaciones, informe, memoria, método, monografía, entre otras) o compuesta (observaciones y experiencias, descripción y análisis, descripción y anuncio, observaciones y reflexiones, estudios y observaciones). Finalmente, se advierten también títulos introducidos por una preposición tematizadora (de, sobre).

En cuanto a los datos cuantitativos, la Tabla 2 representa las frecuencias de títulos con etiquetas (simples y compuestas) respecto a los títulos sin etiquetas y a la totalidad de títulos incluidos en el estudio: 


\begin{tabular}{|l|l|l|l|l|l|l|}
\hline & $\begin{array}{l}\text { Años des } \\
\text { publicación } \\
\text { incluidos }\end{array}$ & $\begin{array}{l}\text { Títulos con } \\
\text { etiqueta } \\
\text { simple }\end{array}$ & $\begin{array}{l}\text { Títulos con } \\
\text { etiquetas } \\
\text { compuestas }\end{array}$ & $\begin{array}{l}\text { Títulos } \\
\text { etiqueta }\end{array}$ & sin & $\begin{array}{l}\text { Núm. total de } \\
\text { títulos }\end{array}$ \\
\hline AHN/ACN & $1799-1804$ & $141(79 \%)$ & $4(2 \%)$ & $33(19 \%)$ & 178 \\
\hline MemoRAC & $1850-1861$ & $55(93 \%)$ & $3(5 \%)$ & $1(2 \%)$ & 59 \\
\hline ASEHN & $1872-1902$ & $88(61 \%)$ & $2(1 \%)$ & $55(38 \%)$ & 145 \\
\hline MemoSEHN & $1903-1920$ & $21(46 \%)$ & $0(0 \%)$ & $25(54 \%)$ & 46 \\
\hline RevRAC & $1904-1920$ & $106(51 \%)$ & $0(0 \%)$ & $103(49 \%)$ & 209 \\
\hline Total & & $411(65 \%)$ & $\mathbf{9 ( 1 \% )}$ & $217(34 \%)$ & $637(100 \%)$ \\
\hline
\end{tabular}

Tabla 1 Títulos con etiquetas y sin etiquetas, resultados cuantitativos

Se observa que la mayoría de los títulos aparecen con alguna etiqueta (420 casos, 66 \%, sumando títulos con etiquetas simples y compuestas) y que el uso de etiquetas compuestas es muy poco frecuente, en comparación con las etiquetas simples (9 casos frente a 411). Además, alrededor de un tercio de títulos (217, 34 \%) aparecen sin etiqueta.

Otro resultado que se desprende de los datos en la Tabla 2 es que la presencia de los títulos con y sin etiquetas varía según la revista. Se observa que, en las dos revistas más tempranas (AHN/ACN y MemoRAC), el número de textos sin etiquetas es considerablemente menor que en la revista publicada en el último tercio del siglo XIX (ASEHN) y en las revistas de inicio del siglo XX (MemoSEHN, RevRAC).

\subsection{Diversidad de las etiquetas y evolución del uso de algunas de ellas}

Respecto a la tipología de las etiquetas, hemos contabilizado 58 etiquetas simples diferentes, lo que indica una variación considerable. Sin embargo, es necesario aclarar que no todas tienen la misma frecuencia de uso. Quince etiquetas aparecen de manera esporádica; se han contabilizado una sola vez en todo el periodo analizado (advertencia, descubrimiento, disertación, indagaciones, razón anotaciones, autógrafo, avance, comentario, elementos, plantilla, reflexión, revisión, sinopsis, trabajo).

En cuanto a las etiquetas más frecuentes, doce de ellas alcanzan una frecuencia de diez o más en todo el periodo analizado (Tabla 3):

\begin{tabular}{|l|l|l|l|l|l|l|l|}
\hline & & $\begin{array}{l}\text { AHN/AC } \\
\text { N }\end{array}$ & $\begin{array}{l}\text { MemoR } \\
\text { AC }\end{array}$ & ASEHN & $\begin{array}{l}\text { MemoSEH } \\
\text { N }\end{array}$ & RevRAC & Total \\
\hline & $\begin{array}{l}1799- \\
1804\end{array}$ & $\begin{array}{l}1850- \\
1861\end{array}$ & $\begin{array}{l}1872- \\
1902\end{array}$ & $1903-1920$ & $\begin{array}{l}1904- \\
1920\end{array}$ & \\
\hline 1 & Discurso & 10 & 26 & & & & 36 \\
\hline 2 & Estudio & & 1 & 6 & 5 & 22 & 34 \\
\hline 3 & Sobre (prepos.) & 2 & & 10 & & 18 & 30 \\
\hline 4 & Observación & 17 & 3 & 6 & 1 & 3 & 30 \\
\hline 5 & Descripción & 24 & 1 & 3 & & & 28 \\
\hline 6 & De (prepos.) & 23 & 1 & 1 & & & 25 \\
\hline
\end{tabular}




\begin{tabular}{|l|l|l|l|l|l|l|l|}
\hline 7 & Catálogo & 1 & 3 & 10 & 2 & 4 & 20 \\
\hline 8 & Ensayo & 1 & 2 & 7 & 3 & 3 & 16 \\
\hline 9 & Nota & 1 & & 4 & 1 & 10 & 16 \\
\hline 10 & Contribución & & & 4 & 4 & 8 & 16 \\
\hline 11 & Memoria & 4 & 11 & & & 15 \\
\hline 12 & Noticia & 6 & 2 & 2 & 1 & & 11 \\
\hline & Total & 89 & 50 & 53 & 17 & 68 & 277 \\
\hline
\end{tabular}

Tabla 2 Las etiquetas más frecuentes (=>10)

Las doce etiquetas cuyas frecuencias se muestran en la Tabla 3 suman 277 títulos, es decir, un $67 \%$ de todos los títulos con etiqueta (420). Se observa, además, que las algunas etiquetas parecen asociarse con una revista y/o un periodo de publicación. Es así, por ejemplo, en el caso de descripción y observación, dos etiquetas muy frecuentes en AHN/ACN. Creemos que, teniendo en cuenta la evolución de las ciencias naturales, ambas etiquetas se relacionan con el tipo de la actividad científica predominante al inicio del siglo XIX.

Por su parte, discurso y memoria son etiquetas típicas para MemoRAC y, en menor medida, para AHN/ACN, mientras que no aparecen en los títulos de las publicaciones más tardías.

El empleo de la etiqueta estudio, en cambio, aumenta a partir del último tercio del siglo XIX y es especialmente frecuente en RevRAC. En cierto modo, esto se podría valorar como una distribución complementaria en relación con memoria. Apenas contamos con casos de estudios en las revistas más tempranas (un solo caso en MemoRAC), y su frecuencia aumenta a la vez que de los índices de las revistas analizadas desaparecen las memorias. Parece, por tanto, que inicialmente fueron las memorias los textos científicos o artículos "por excelencia» o que esta fue la denominación genérica para los textos incluidos en las revistas del siglo XIX. Más tarde, estudio parece apropiarse esta función, mientras que memorias se convierten en trabajos más extensos, monográficos.

Para comprobar si esta intuición se sustenta con datos lexicográficos, hemos realizado búsquedas en diccionarios históricos de la Real Academia Española (RAE) ${ }^{15}$ y en algunos diccionarios no académicos del siglo XIX'16. En cuanto a la memoria como tipo de texto, en los diccionarios académicos se define como disertación hasta finales del siglo XIX:

\section{memoria}

'Lo mismo que DISERTACION' (Academia Usual, 1817); 'Disertación' (Academia Usual, 1884)

\section{disertación}

'Discurso en que se proponen las razones a favor de alguna opinión y se impugnan las contrarias' (Academia Usual, 1843)

\footnotetext{
${ }^{15}$ Para ello, hemos utilizado la herramienta digital Mapa de diccionarios académicos, accesible en línea: http://web.frl.es/ntllet. 16 Los diccionarios se han consultado en la aplicación en línea de Nuevo Tesoro Lexicográfico de la RAE, http://buscon.rae.es/ntlle/SrvitGUILoginNtlle. 
Un cambio interesante se observa al inicio del siglo XX, cuando la definición se extiende a estudio:

\section{memoria}

‘8. Estudio, o disertación escrita, sobre alguna materia' (Academia Usual, 1914).

El propio estudio se documenta como tipo de texto ya a lo largo del siglo XIX, haciendo referencia a estudios (ant.) en el sentido de '(o)bras, escritos, trabajos literarios' (Salvá, 1846) u '(o)bras, escritos, tratados, etc.' (Domínguez, 1853), pero es a inicios del siglo XX (1917) cuando encontramos una definición de estudio que lo describe como un trabajo escrito y sugiere su carácter científico:

\section{estudio}

'Trabajo, investigación, disquisición, disertación, tratado, ensayo’ (Alemany y Bolufer, 1917)

\section{estudio}

‘2. Obra en que un autor estudia y dilucida una cuestión’ (Academia Usual, 1925)

Creemos que la incorporación de estudio en la definición de memoria refleja los cambios acontecidos en la comunicación científica.

Aparte de las observaciones hechas acerca de algunas etiquetas utilizadas en los títulos de los artículos, resulta interesante comentar también el uso de las preposiciones tematizadoras de y sobre sin presencia de otra palabra descriptiva, por ejemplo: «De la utilidad del Cacahuete ó Arrachis hypogea», "Sobre la reproducción del Rhinoderma Darwini»). Se ve un claro cambio de preferencia en el uso de una u otra preposición. Inicialmente, en las publicaciones más tempranas (AHN/ACN), predomina el uso de la preposición de, mientras que esta preposición a modo de palabra introductora del título ya no se registra en las publicaciones de los inicios del siglo XX; en RevRAC aparecen únicamente títulos encabezados por la preposición sobre, mientras que en la MemoSEHN, ninguna de las dos. De acuerdo con Gross y otros (2002: 83), las preposiciones tematizadoras aparecían a menudo en los títulos de los artículos científicos europeos del siglo XVIII como una especie de convención cultural: "They are a form of social signaling, like the handshake in Western cultures: they mark an entrance into the domain of study, in this case, science». Con ello se demuestra que el género de artículo científico español seguía, en buena manera, las tendencias de la ciencia internacional.

\subsection{Uso de las etiquetas vs prescripciones editoriales de las revistas}

En el apartado 3 hemos citado algunos documentos institucionales publicados en las revistas analizadas en los que se aludía al tipo de textos que se incluirían en cada una de ellas, según el proyecto editorial. Asimismo, queremos comparar esta información con los resultados de nuestra exploración de los índices para comprobar si, en la práctica, es cierto que las revistas incluyen de manera predominante un tipo de textos específico o no. Recordemos, en primer lugar, con qué palabras se definían los textos que se publicarían en cada revista, según los documentos institucionales citados en el apartado 3 :

AHN/ACN: descubrimientos, memorias 
MemoRAC: resumen, memoria, informe, elogio

ASEHN: catálogo, crítica, descripción, monografía, noticia

MemoSEHN: estudio, catálogo, trabajo, monografía

En este momento consideramos estas palabras como una especie de «etiquetas prescritas» por cada institución, aunque somos conscientes que en algunos casos puede tratarse simplemente de palabras descriptivas de los contenidos de las revistas, tal como fueron planteadas (este sería seguramente el caso de descubrimientos).

Si comparamos estas palabras con las etiquetas más frecuentes en los índices de las revistas (véase la Tabla 3), comprobamos que cinco etiquetas coinciden: estudio, descripción, catálogo, memoria y noticia. Cabe apuntar que, aunque con menos frecuencia, los otros tipos de textos mencionados en los documentos institucionales también se registraron al menos una vez en la muestra de títulos analizada, tal fue el caso de descubrimiento, informe, monografía y trabajo. Los únicos tipos de textos mencionados por los editores de las revistas que no aparecen a modo de etiquetas en la muestra los títulos son elogio, resumen y crítica.

En esta parte del estudio concluimos que la coincidencia de las cinco etiquetas frecuentes en ambos conjuntos de datos (es decir, documentos institucionales y los títulos) muestra que, efectivamente, los editores prescribían, hasta cierto punto, el tipo de textos que aparecerían en las páginas de las revistas. Además, parece que los autores de los artículos tenían conciencia acerca de esta política editorial. Sin embargo, la diversidad de las etiquetas encontradas en los títulos supera el número de tipos de textos mencionados en los documentos institucionales (58 etiquetas diferentes frente a 12 tipos de texto mencionados en los documentos). Así pues, se observa que la invención de los autores con este respecto superaba con creces las previsiones de los editores. Suponemos que la multitud de etiquetas diferentes ( $\mathrm{y}$, en muchos casos, muy poco frecuentes) es un buen indicio de que nos encontramos ante un periodo de vacilaciones. Sugieren que la estandarización del formato del artículo científico estaba en el proceso de convencionalizarse. A lo largo del mismo, algunas de las etiquetas no superaron la prueba del tiempo (descripción), mientras que otras se convirtieron en ejemplares hacia el final del periodo que hemos analizado (estudio).

\section{CONCLUSIONES}

Los datos que hemos consultado demuestran una interesante tendencia en la nomenclatura de los artículos científicos publicados en las revistas institucionales en el periodo comprendido entre 1799 y 1920 . Se trata del uso frecuente de etiquetas o palabras descriptivas del tipo de texto que pretenden especificar su carácter. Parece que, en algunos casos, se trata de una práctica relacionada con las prescripciones editoriales, puesto que en los documentos institucionales encontramos mención de los tipos de textos que se publicarían en sus revistas. Sin embargo, hemos visto que la invención de los propios autores de los textos en este aspecto supera las recomendaciones institucionales, ya que el número de etiquetas diferentes (58) utilizadas en los títulos es casi cinco veces mayor que en el número de nombres de textos que sugeridos en los documentos institucionales. A pesar de esta gran variedad de etiquetas, solamente unas pocas (12) registran uso frecuente a lo largo de todo el periodo, mientras que muchas otras se utilizan de manera muy esporádica. 
En cuanto a la evolución temporal del uso de las etiquetas más frecuentes, este no es estable en todas las revistas ni a lo largo de todo el periodo. El uso de la etiqueta estudio, por ejemplo, aumenta en las revistas más modernas, mientras que desaparecen de ellas las memorias. Los datos lexicográficos parecen corroborar la intuición de que estudio se constituye como un prototipo de texto científico al inicio del siglo XX, a la vez que sustituye en esta función a memoria.

Hemos observado también el uso de las preposiciones tematizadoras como etiquetas, lo cual concuerda con las tendencias observadas en los artículos de los siglos XVIII y XIX en otras lenguas. Las preposiciones también se ven afectadas por el paso del tiempo: mientras que al inicio del siglo XIX era predominante el uso de de, esta preposición es sustituida por sobre al principio del siglo XX.

Por su parte, los títulos que no incluyen ninguna etiqueta constituyen un $34 \%$ de todos los títulos analizados, pero se trata de un número que varía entre distintas revistas y, también en el tiempo. Se ha observado un aumento de este tipo de títulos a partir del último tercio del siglo XIX.

Creemos que los resultados de este estudio, aunque no trazan la evolución del género del artículo científico de manera exhaustiva, ofrecen pistas sobre la misma. Permiten ver una progresiva especialización en cuanto a los contenidos de las publicaciones científicas, reflejada en el uso de las etiquetas que aluden a la actividad científica realizada en cada momento histórico (observaciones - descripciones - estudios, etc.)

\section{BIBLIOGRAFÍA}

AlgabA, Antonio (2000): «La difusión de la innovación. Las revistas científicas en España 1760-1936», Scripta Nova. Revista Electrónica de Geografía y Ciencias Sociales, 69 (17), Universidad de Barcelona, http://dx.doi.org/10.1344/sn2000.4.235.

BANKS, David (2008): Development of Scientific Writing: Linguistic Features and Historical Context, Londres, Equinox Publishing Ltd.

BAZERMAN, Charles (1988): Shaping Written Knowledge: The Genre and Activity of the Experimental Article in Science, Madison, Wisconsin, WAC Clearinghouse Landmark Publications in Writing Studies/University of Wisconsin Press.

Bosch Carrera, M. ${ }^{a}$ Dolors (1992): «Los inicios de las revistas especializadas en España", Hispania, 180(52), 263-277.

Correll DoméneCH, M. y V. NAVARRO Brotons (2004), «Prensa y periodismo científico en España», en Fernández Sanz, J. J., coord., Doce calas en la historia de la prensa española especializad, Guadalajara, Editores del Henares, 53-82.

Gross, Alan G. y otros (2002): Communicating Science. The scientific article from the 17th century to the present, Oxford, Oxford University Press.

GONZÁlEZ GONZÁLEZ, Francisco Javier (1999): «Publicaciones periódicas científicas (siglos
XVII, XVIII y XIX): Fondos conservados en la biblioteca del Real Instituto y Observatorio de la Armada», Cuadernos de Ilustración y Romanticismo, 7, 75-83.

HYLAND, Ken (2009): Academic discourse, Londres, Continuum International Publishing.

LÓPEZ PIÑERO, José M. (1992): «Introducción», en López Piñero, J. M., ed., La ciencia en la España del siglo XIX, Madrid, Marcial Pons, 1118.

LÓPEZ PIÑERO, José M. y María Luz LóPEZ TeRRADA (1990): Bibliographia medica hispanica, 14751950. Vol. VIII. Revistas, 1736-1950, Valencia, Instituto de Estudios Documentales e Históricos sobre la Ciencia de la Universidad de Valencia.

McCLELLAN, James. E. (1979): «The scientific press in transition: Rozier's Journal and the scientific societies in the 1770s", Annals of Science, 36(5), 425-449.

PARODI, Giovanni y otros (2009): «El Corpus PUCV2006 del Español: identificación y definición de los géneros discursivos académicos y profesionales», Lingüística: artículos y monografías, Literatura y Lingüística, 75-101.

SWALES, John. M., ed. (2007 [1990]): Genre Analysis. English in academic and research settings, Cambrigde, Cambridge University Press. 
SwALES, John M. (2004): Research Genres. Exploration and Applications, Cambridge, Cambridge University Press.

Ten Ros, Antonio y María Cell Aragón (1996): Catálogo de las revistas científicas y técnicas publicadas en España durante el s. XIX, Cuadernos Valencianos de Historia de la Medicina y de la Ciencia, 52, Valencia, Instituto de Estudios Documentales e Históricos sobre la Ciencia/Universitat de Valencia/CSIC.

VAlle, Ellen (2004): «'A nice and accurate philosopher': Interactivity and Evaluation in a Historical Context», en Del Lungo Camiciotti, G. y E. Tognini Bonelli eds., Linguistic Insights, 15, Academic Discourse-New Insights into Evaluation, Bern, Peter Lang, 55-80. 